\title{
Extremely uniform lasing wavelengths of InP microdisk lasers heterogeneously integrated on SOI
}

\author{
Pauline Mechet, Thijs Spuesens, Dries Van Thourhout, \\ Gunther Roelkens, Geert Morthier \\ Photonics Research Group, Ghent University - imec \\ Department of Information Technology (INTEC) \\ Sint-Pietersnieuwstraat 41 \\ 9000 Ghent, Belgium
}

\begin{abstract}
A standard deviation in lasing wavelength lower than $500 \mathrm{pm}$ is characterized on nominally identical and opticallypumped microdisk lasers, heterogeneously integrated on the same SOI circuit. This lasing wavelength uniformity is obtained using electron-beam lithography.
\end{abstract}

Keywords- Microdisk Lasers, Silicon Photonics, On-chip Laser Sources, All-optical Signal Processing, Cascadability

\section{INTRODUCTION}

Heterogeneous integration based on die-to-wafer bonding of III-V compound semiconductors on SOI waveguide circuits has been demonstrated as a promising platform for integrated active devices on a silicon chip. Microdisk lasers have attracted considerable attention for their potential as compact and lowthreshold coherent light sources. They support whispering gallery modes that propagate at the periphery of the microdisk cavity. Such a compact laser has been used to demonstrate an all-optical flip-flop memory element [1] as well as a multiwavelength laser source [2]. In order to increase the cascadability of the all-optical system, i.e. the number of identical gates or logic blocks that can be concatenated without losing signal integrity, the wavelengths of the microdisk lasers must be aligned to each other. To some extent, it is possible to control the lasing wavelength of the microdisks by processing the devices with high accuracy patterning tools, e.g. electronbeam lithography.

In this work, we study the room temperature and continuous wave laser emission of InP-based microdisk lasers, fabricated using e-beam lithography, heterogeneously integrated and evanescently coupled to SOI waveguides.

\section{DEVICE DESIGN AND FABRICATION}

The devices studied in this work are schematically depicted in Fig 1. Two optical levels can be identified. The lowest level consists of narrow single mode SOI waveguides embedded in $\mathrm{SiO}_{2}$ (with a sweep in width and a height of 220nm). The SOI waveguides are fabricated in a CMOS fab using $193 \mathrm{~nm}$ deep ultra-violet lithography. The upper level consists of a $583 \mathrm{~nm}$ thick InP-based membrane with an InP-based tunnel junction and three embedded InAsP quantum wells (QWs) emitting

\author{
Fabrice Raineri, Alexandre Bazin, Paul Monnier, \\ Isabelle Sagnes, Rama Raj \\ Laboratoire de Photonique et Nanostructures (CNRS) \\ Route de Nozay, 91460 Marcoussis, France \\ Philippe Regreny \\ Institut des Nanotechnologies de Lyon INL-UMR5270 (CNRS) \\ Université et Ecole Centrale de Lyon, 69134 Ecully, France
}

around 1530nm. The two levels are separated by a thin transparent layer $(140 \mathrm{~nm})$ of a low refractive index material $\left(n=1.54\right.$ for DVS-BCB) and $100 \mathrm{~nm}$ of $\mathrm{Al}_{2} \mathrm{O}_{3}(\mathrm{n}=1.7)$, allowing evanescent coupling to the underlying waveguide (WG). The designed microdisk lasers have a diameter between 6 and $7.5 \mu \mathrm{m}$. For the fabricated sample under study, the design of the passive level consists of 5 sections of nominally identical waveguides with a given width $w$, as depicted on Fig. 1. The smallest value of $w$ is $300 \mathrm{~nm}$. $w$ increases with a $50 \mathrm{~nm}$-step from one section to another. The largest waveguide width on the sample is then $500 \mathrm{~nm}$. The offset between the edge of the microdisk and the edge of the waveguide is kept constant within one section.

(a)

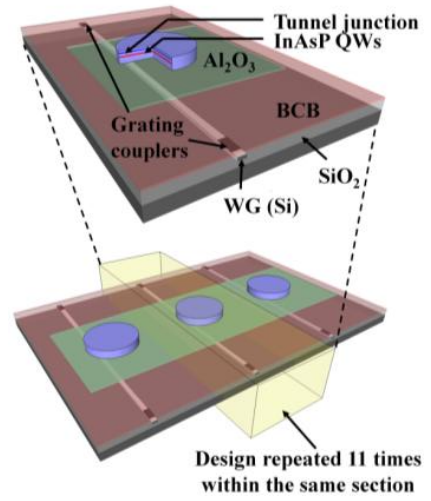

Fig. 1. Schematic of the sample. (a) Microdisk laser structure coupled to underlying waveguide. (b) Array of nominally identical microdisk lasers.

The device fabrication relies on the adhesive bonding of an unprocessed MBE-grown InP-based heterostructure onto the SOI with the use of the planarizing polymer DVS-BCB. Alignment markers for subsequent processing are defined in the $\mathrm{Si}$ on the same mask layer and relative to the waveguide structures [3]. This allows the accurate electron-beam alignment of microdisk lasers with respect to the waveguides.

Microdisks are patterned into the bonded InP material following one single electron-beam exposure [4] and according to a design generated with a $2.5 \mathrm{~nm}$ writing resolution. To achieve this, a $300 \mathrm{~nm} \mathrm{Si}_{3} \mathrm{~N}_{4}$ hard mask is deposited on top of 
the epitaxy, and a $300 \mathrm{~nm}$-thick layer of ma-N negative resist is spin-coated on top of it. The microdisks are then automatically exposed in the ma-N layer. Following the development of the resist, the pattern is transferred into the $\mathrm{Si}_{3} \mathrm{~N}_{4}$ mask and the microdisks are etched in the III-V membrane using ICP etching. The $\mathrm{Si}_{3} \mathrm{~N}_{4}$ mask is removed by a RIE process. SEM measurements show that the microdisk lasers are aligned with respect to the SOI wires with an alignment accuracy better than $40 \mathrm{~nm}$. This level of accuracy enables reproducibility in the fabrication and a close control of the evanescent coupling.

\section{LASING WAVELENGTH DEVIATION OF NOMINALLY IDENTICAL MICRODISK LASERS ON THE SAME CHIP}

Microdisks with nominally identical designs and coupled to waveguides with the same width are studied under optical pumping. The CW light of a laser emitting at $980 \mathrm{~nm}$ is focused on the top surface of the microdisks using a singlemode fiber under a $10^{\circ}$ angle. The light emitted from the microdisk lasers couples to the TE mode of the underlying waveguide. Another fiber positioned above one grating coupler collects the laser emission of the microdisks also under a $10^{\circ}$ angle. Working at constant pump power for every device of every section, the spectrum of each microdisk above threshold is recorded on an optical spectrum analyzer with a 100 -pm resolution. Table $\mathrm{I}$ is a summary of the results for 5 different sections on the same sample. For instance, a standard deviation of $0.37 \mathrm{~nm}$ on the lasing wavelength of 9 nominally identical $7.5-\mu \mathrm{m}$ diameter microdisks and coupled to $450-\mathrm{nm}$ wide waveguides is measured. The spectra from the microdisk lasers are plotted together on Fig. 2. From this characterization, we demonstrate that a standard deviation in lasing wavelength of nominally identical devices on the same chip lower than $500 \mathrm{pm}$ is achievable.

TABLE I. Standard deviation in lasing wavelength of microdisk lasers on the same die.

\begin{tabular}{cccc}
\hline \hline $\begin{array}{c}\text { Disk } \\
\text { diameter }\end{array}$ & $\begin{array}{c}\text { Number } \\
\text { of disks }\end{array}$ & $\begin{array}{c}\text { WG } \\
\text { width }\end{array}$ & $\begin{array}{c}\text { Standard deviation in } \\
\text { lasing wavelength }\end{array}$ \\
\hline $7.5 \mu \mathrm{m}$ & 10 & $500 \mathrm{~nm}$ & $\mathbf{0 . 4 4} \mathbf{~ n m}$ \\
$7.5 \mu \mathrm{m}$ & 9 & $450 \mathrm{~nm}$ & $\mathbf{0 . 3 7} \mathbf{~ n m}$ \\
$7 \mu \mathrm{m}$ & 7 & $400 \mathrm{~nm}$ & $\mathbf{0 . 6 7} \mathbf{~ n m}$ \\
$7 \mu \mathrm{m}$ & 11 & $350 \mathrm{~nm}$ & $\mathbf{0 . 4 8} \mathbf{~ n m}$ \\
$6 \mu \mathrm{m}$ & 11 & $300 \mathrm{~nm}$ & $\mathbf{0 . 4 4} \mathbf{~ n m}$ \\
\hline \hline
\end{tabular}

A 500pm standard deviation in lasing wavelength for 7.5$\mu \mathrm{m}$ diameter microdisks is related to a deviation in the diameter of the processed devices as low as $2.3 \mathrm{~nm}$.

Under electrical pumping, a maximal tuning efficiency of the lasing wavelength of 7.5- $\mu \mathrm{m}$ diameter microdisk lasers of $0.35 \mathrm{~nm} / \mathrm{mW}$ has been achieved by electrically heating a III-V semiconductor arc closely located to the microdisk cavity [5]. This device is used for compensating wavelength variations resulting from fabrication. Such a technology could very well be implemented to compensate the standard deviation characterized in this paper, with low additional power consumption.

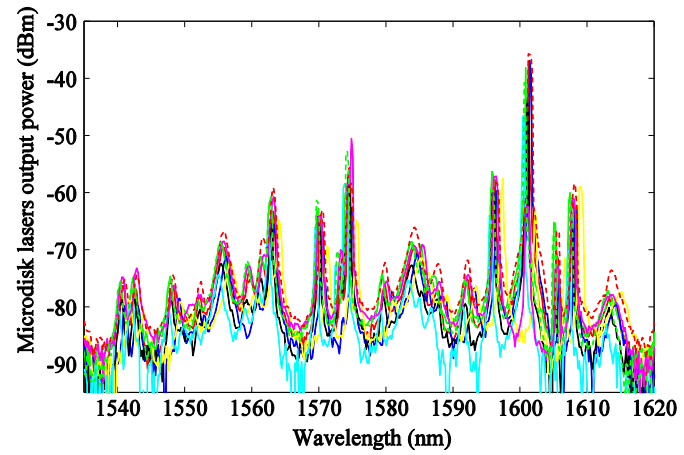

Fig. 2. Spectra of 9 nominally identical microdisk lasers processed with e-beam lithography and optically pumped at $980 \mathrm{~nm}$. The standard deviation in peak lasing wavelength is $0.37 \mathrm{~nm}$.

We also evaluate the deviation in pump power needed to achieve lasing. Six out of seven nominally identical microdisk lasers (WG width of $500 \mathrm{~nm}$ ) exhibit single-mode operation for a standard deviation in pump power of $1.73 \mathrm{~dB}$.

\section{CONCLUSION}

Detection of electron beam alignment markers has allowed the very accurate definition of microdisk lasers with respect to Si wire waveguide. The achievable and reproducible standard deviation in their peak lasing wavelengths is lower than $500 \mathrm{pm}$ on the same chip. The processing optimizations open the door to a more accurate control of the efficiency of the coupling between the microdisk and the SOI. The fabrication of complex functionalities for all-optical signal processing requiring cascading several microdisk lasers on the same chip is now made possible.

\section{ACKNOWLEDGMENT}

We thank Rémy Braive for the ICP etching of the microdisk lasers and the ICT FP7 European Projects HISTORIC and WADIMOS.

\section{REFERENCES}

[1] L. Liu, R. Kumar, K. Huybrechts, T. Spuesens, G. Roelkens, E.-J. Geluk, T. de Vries, P. Regreny, D. Van Thourhout, R. Baets, and G. Morthier, "An ultra-small, low-power, all-optical flip-flop memory on a silicon chip," Nature Photonics 4(3), 182-187 (2010).

[2] J. Van Campenhout, L. Liu, P. R. Romeo, D. Van Thourhout, C. Seassal, P. Regreny, L. Di Cioccio, J.-M. Fedeli, and R. Baets, "A Compact SOIIntegrated Multiwavelength Laser Source Based on Cascaded InP Microdisks," IEEE Photonics Technol. Lett. 20(16), 1345-1347 (2008).

[3] T. J. Karle, Y. Halioua, F. Raineri, P. Monnier, R. Braive, L. Le Gratiet, G. Beaudoin, I. Sagnes, G. Roelkens, F. van Laere, D. Van Thourhout, and R. Raj, "Heterogeneous integration and precise alignment of InPbased photonic crystal lasers to complementary metal-oxide semiconductor fabricated silicon-on-insulator wire waveguides," J. Appl. Phys. 107, 063103 (2010).

[4] Y. Halioua, T. Karle, F. Raineri, P. Monnier, I. Sagnes, R. Raj, G. Roelkens, and D. Van Thourhout, "Hybrid InP-based photonic crystal lasers on silicon on insulator wires," Appl. Phys. Lett. 95(20), 201119 (2009).

[5] L. Liu, T. Spuesens, G. Roelkens, D. Van Thourhout, P. Regreny, and P. Rojo-Romeo, "A thermally tunable microdisk laser built on a IIIV/Silicon-on-insulator heterogeneous integration platform," IEEE Photonics Technol. Lett 22, 1270 (2010). 